OPEN ACCESS

Edited by:

Zsolt J. Balogh,

The University of Newcastle, Australia

Reviewed by:

Seth Michael Tarrant,

Hunter New England Health, Australia

Holly Tyler-Paris Pilson,

Wake Forest Baptist Medical Center,

United States

*Correspondence:

Pierre Navarre

navarre.pierre@gmail.com

Specialty section: This article was submitted to

Orthopedic Surgery,

a section of the journal

Frontiers in Surgery

Received: 25 October 2020 Accepted: 14 December 2020

Published: 28 January 2021

Citation:

Russ MK, Navarre $P$ and Stevens JP (2021) An Alternative Site for Pin Placement in External Fixation of Pelvic Fractures: Lateral Posterior Pelvic External Fixator Surgical Technique. Front. Surg. 7:621125 doi: 10.3389/fsurg.2020.621125

\section{An Alternative Site for Pin Placement in External Fixation of Pelvic Fractures: Lateral Posterior Pelvic External Fixator Surgical Technique}

\author{
Matthias K. Russ ${ }^{1,2,3}$, Pierre Navarre ${ }^{1,4,5 *}$ and Jarrad P. Stevens ${ }^{1,6}$ \\ ${ }^{1}$ Department of Orthopaedic Surgery, Alfred Hospital, Melbourne, VIC, Australia, ${ }^{2}$ Cabrini Hospital, Orthopaedic Surgery, \\ Melbourne, VIC, Australia, ${ }^{3}$ Department of Surgery, Monash University, Melbourne, VIC, Australia, ${ }^{4}$ Department of \\ Orthopaedic Surgery, Southland Hospital, Invercargill, New Zealand, ${ }^{5}$ Department of Surgical Sciences, University of Otago, \\ Dunedin, New Zealand, ${ }^{6}$ Knox Orthopaedic Group, Knox Private Hospital, Melbourne, VIC, Australia
}

Introduction: The application of an external fixator for unstable pelvic fractures is an important component of many resuscitation protocols. Moreover, certain pelvic fractures may be treated with an external fixator without requiring further internal fixation. We report our initial clinical results with an alternate pelvic external fixator site, the lateral posterior external fixator (LPEF), and describe the surgical technique.

Methods and Materials: From 2010 to 2013, we identified 27 consecutive patients (mean age 44.6 years, range 18-80 years) treated by the same surgeon (MKR) with an LPEF in a level 1 trauma center. Retrospective data collection included mechanism of injury, surgical interventions, and complications.

Results: The LPEF was used in 16 patients as acute pelvic stabilization and converted at a median of 2 days (interquartile range 1-3.5) to internal fixation, whereas in 10 patients, it was used as definitive treatment and removed at a median of 48 days (interquartile range 37-64). One patient died on day 14 , secondary to his severe closed head injury. The only surgical complications were two wound infections (20\%,2/10 in the group of definitive LPEFs), which resolved without sequelae after the removal of the LPEF (at 36 and 50 days) and antibiotics, one case of loss of fixation leading to the removal of the LPEF at 71 days, and one patient who had hypergranulating external fixator sites and eventually healed without any cutaneous sequelae. All fractures consolidated in a good position.

Discussion: The described techniques of pelvic external fixation include the anterosuperior (iliac wing), supra-acetabular (anteroinferior), and subcristal (anterior superior iliac spine) insertion sites. The reported infection rates in definitive pelvic fracture treatment range from 20 to $40 \%$. Due to the localization of the insertion sites, the lateral femoral cutaneous nerve is potentially at risk with the last two techniques. On the other hand, the LPEF insertion site is quite safe, as it is anatomically far from any nerves and the inguinal region, and allows easy access for laparotomy. The results in this series suggest that the lateral posterior pelvic external fixator technique is an alternative to previous techniques with a low risk of complications.

Keywords: pelvic fracture, polytrauma, external fixation, damage-control orthopedics, lateral posterior external fixator 


\section{INTRODUCTION}

Application of external fixation for major pelvic trauma is an important component of many resuscitation protocols, with the goal of stabilizing the pelvis to control hemorrhage, decrease blood transfusions rates, and improve survival rates (1-5). The procedure should be simple, quick, have minimal risk of complications, and be reproducible for surgeons who may rarely require its use in regional settings. Moreover, certain pelvic fractures may be treated with an external fixator without requiring further internal fixation.

The surgical anatomy of three techniques for anterior pelvic external fixation has been well-described, including anterosuperior (iliac wing), supra-acetabular or anteroinferior (anterior inferior iliac spine), and subcristal [anterior superior iliac spine (ASIS)] insertion sites (6). Associated risks of complications are well-recognized, including pin malposition, loss of fixation, neurovascular injury, and infection (6-10). We describe the initial clinical results using a lateral posterior pin site entry technique, which we have named the lateral posterior external fixator (LPEF). Using the tuberous portion of the lateral iliac crest as an identifiable landmark, we can generate a lateral entry point and directing posteriorly, thus positioning our pins further away from the groin crease and skin folds caused by the sitting position (Figure 1). The senior author (MKR) has developed and used this technique since 2010, and it is currently his technique of choice in many pelvic fracture configurations, both for initial stabilization and definitive treatment.

We have received approval from the Alfred Hospital Ethics Committee $(466 / 13)$ for this study.

\section{METHODS AND MATERIALS}

We have identified 27 consecutive patients who have been treated with an LPEF by a single surgeon (MKR) between 2010 and 2013. The patients were identified by index codes from medical records at The Alfred, a level 1 referral trauma center in Victoria, Australia. Inclusion criteria were patients older than 18 years of age treated with an LPEF for acute treatment of a pelvic ring injury. Exclusion criteria were patients who had a different type of pelvic external fixator, other than the LPEF. We proceeded to a retrospective medical records review and collected data relative to injury mechanism, surgical interventions, complications, including infection (superficial pin site infection defined as cellulitis and purulent discharge responding to dressings and pin site care or removal of external fixator without evidence of osteomyelitis, deep infection defined as osteomyelitis, or deep collection), cutaneous complications (such as hypergranulating wounds), iatrogenic fracture, gross malposition of the external fixator (missing the iliac tuberosity on postoperative radiographs), loss of fixation, nerve or vascular injury, and demographic data. Lateral femoral cutaneous nerve injury was defined by clinical

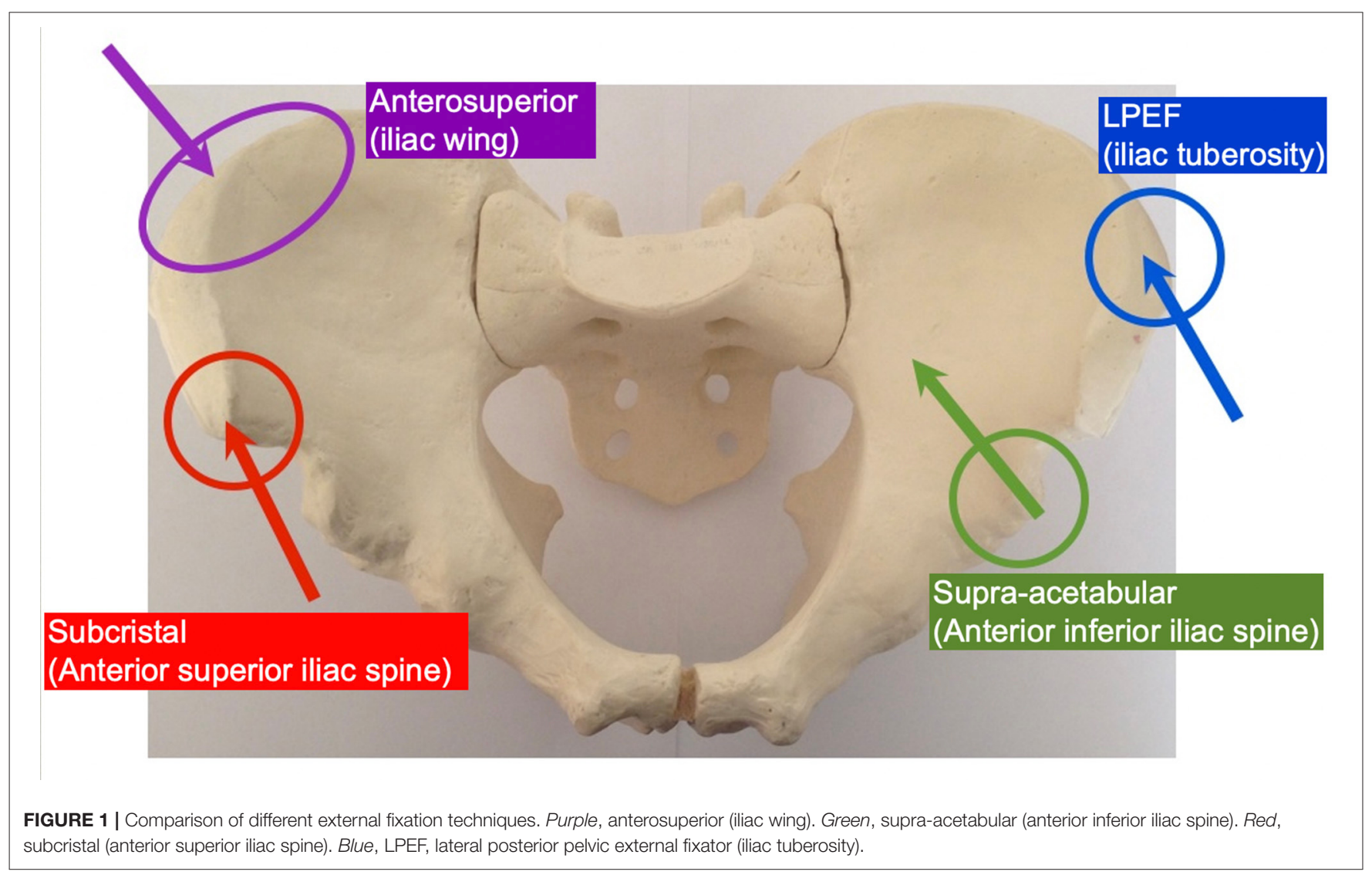




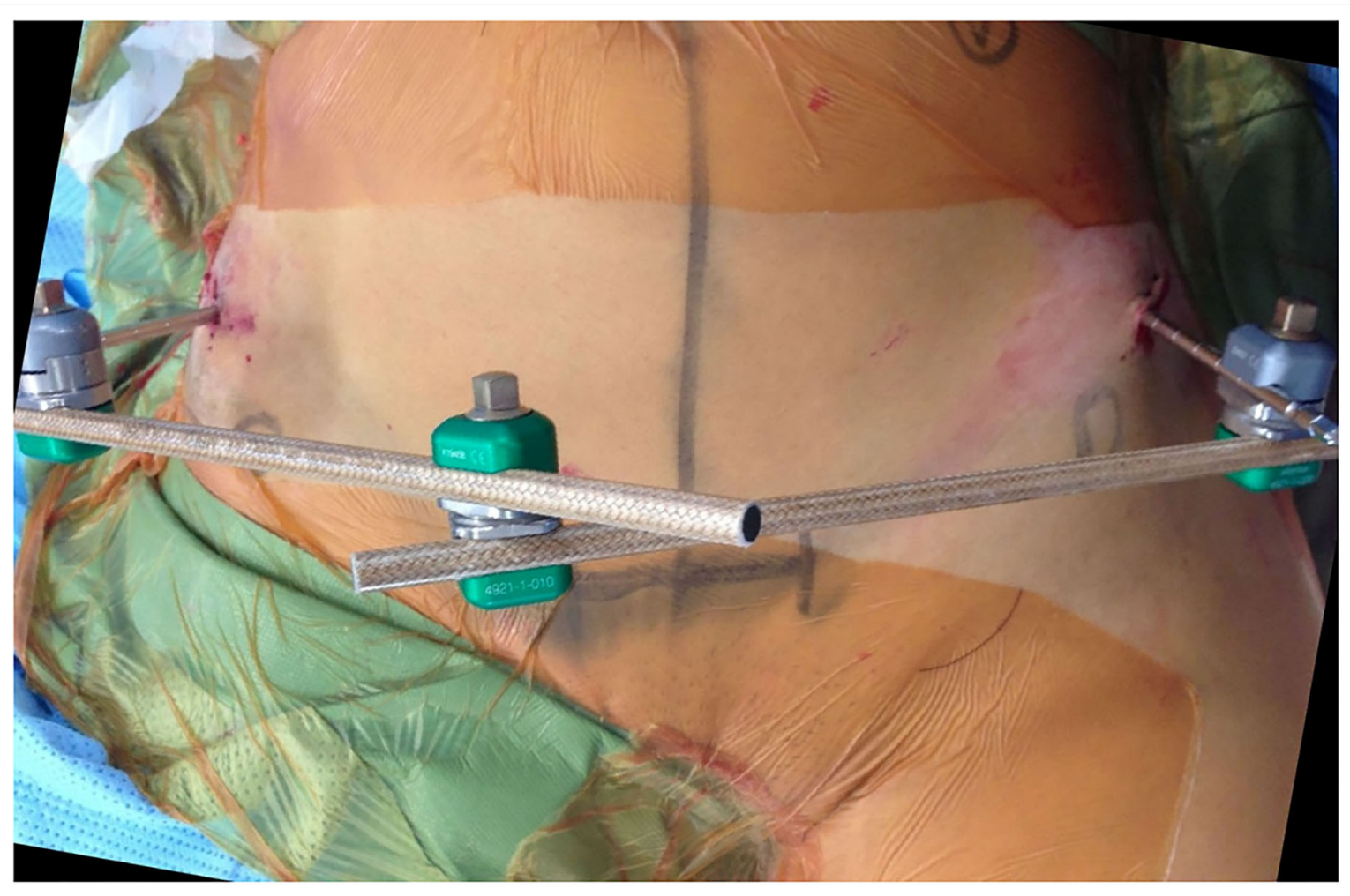

FIGURE 2 | LPEF technique, view from above. Pin insertion posterolateral to the ASIS (marked by circles) in slight lateral to medial and caudal to cranial direction.

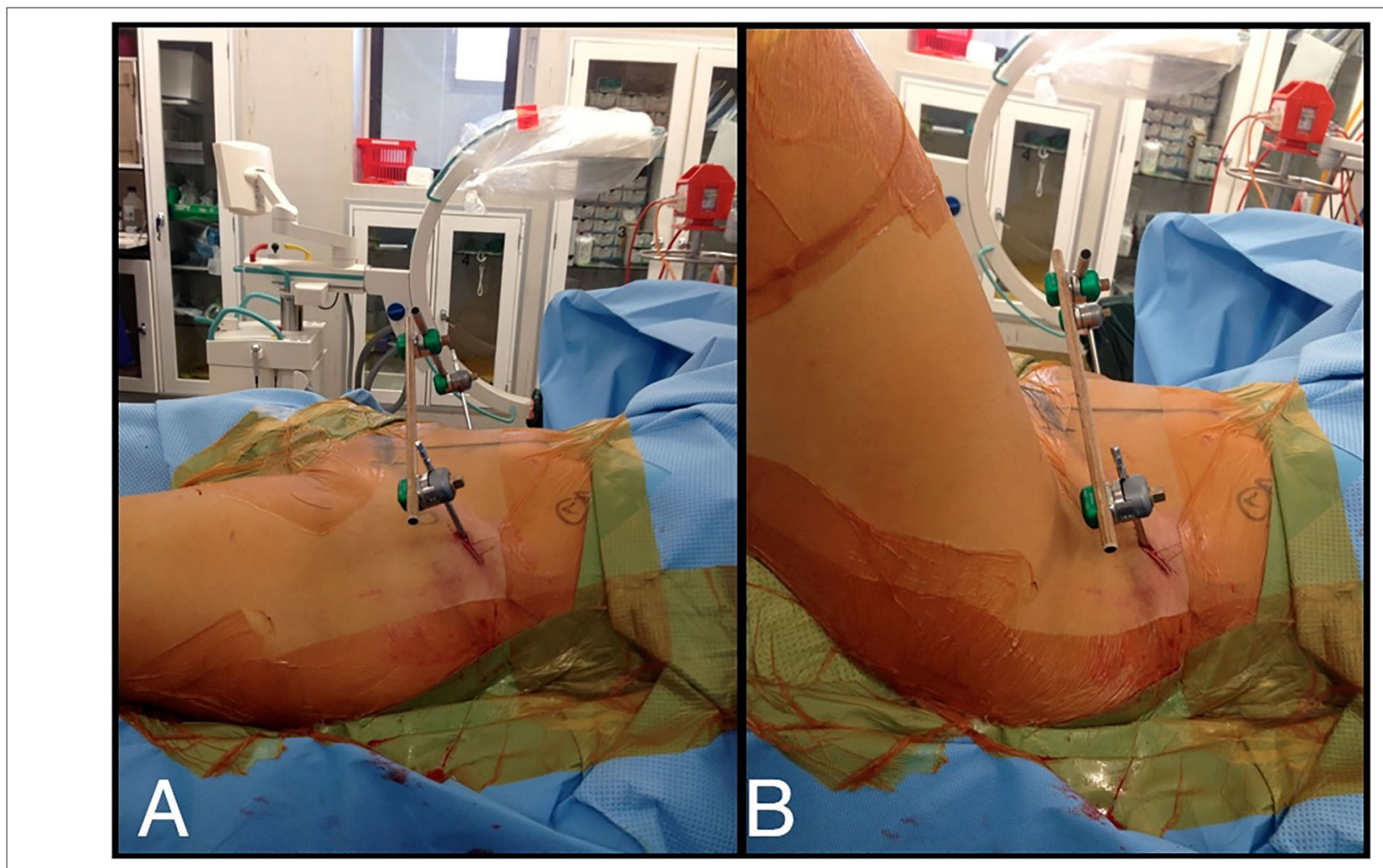

FIGURE 3 | (A) LPEF technique, view from side. (B) Flexion of the hips not hindered by LPEF pins and flexion creases far from LPEF insertion site. 


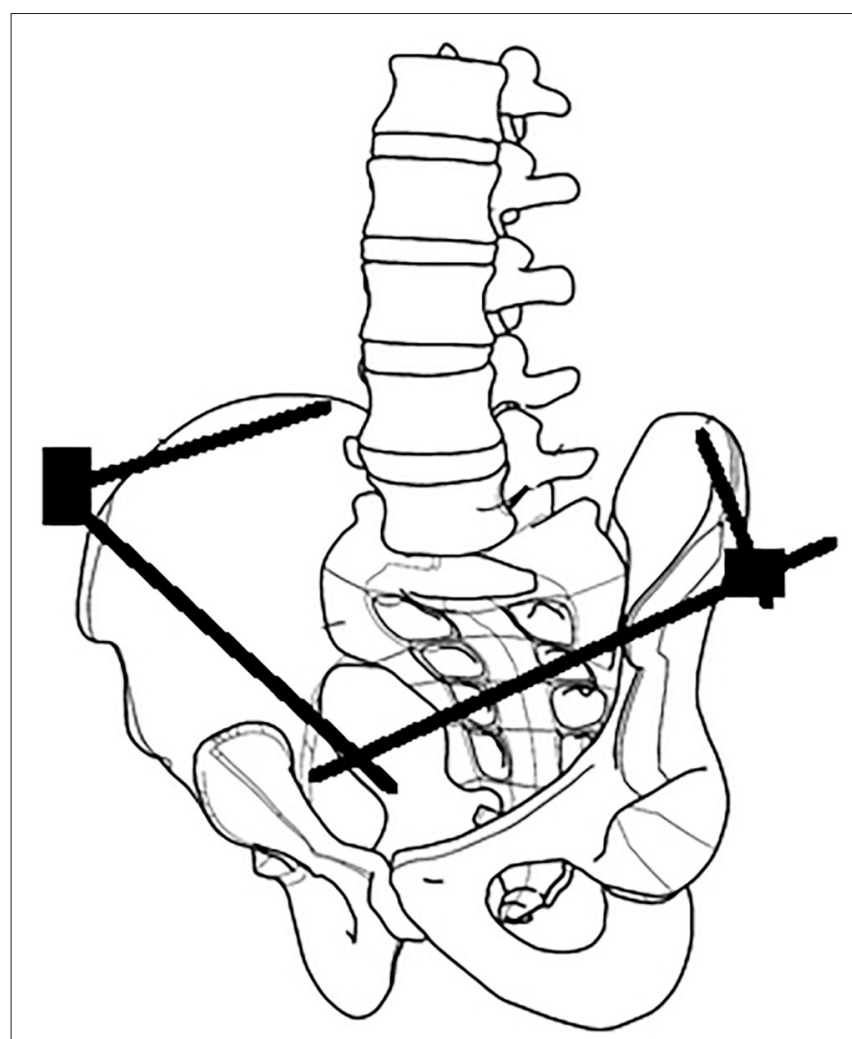

FIGURE 4 | Line-drawing image representing the position of the LPEF.

examination of numbness or loss of sensation in the anterolateral proximal thigh. Descriptive statistics for all sociodemographic variables and injury factors will be reported using mean and range for normally distributed continuous data, mean and interquartile range (IQR) for continuous data that were not normally distributed, and proportions for categorical variables. All analyses were performed using Stata Version 13.

\section{Surgical Technique}

The patient is positioned supine on a radiolucent table, our preference being the Jackson table. This allows easy application of the pelvic external fixator, external or internal fixation of any fractured limbs, and access for the image intensifier. Draping of the patient should allow access to the entire abdomen down to the level of the table and the proximal thighs, excluding the genital area from the sterile field. This allows access for laparotomy as required and access for added external fixator sites as required. If there are fractures of the lower limbs, they can be included in the sterile field in the same setting to minimize operative time.

Identifiable landmarks include the ASIS and the iliac crest, which is palpated posterolaterally from the ASIS. The tuberous portion of the iliac crest is palpated as the iliac crest curves posteriorly and medially. These landmarks are outlined with a surgical marking pen (Figure 2). A 2-cm incision is made overlying the tuberous portion of the iliac crest in the superolateral to inferomedial direction, allowing incision extension as required. An artery clip is used to bluntly dissect the subcutaneous tissues and reach the tuberous portion of the iliac crest. This is a percutaneous technique; however, two small retractors can aid in visualization as required. The periosteum is carefully incised in its center with a 15-blade scalpel or electrocautery. A 5-mm by 150 or 180 -mm partially threaded selfdrilling and a self-tapping pin is used with a soft-tissue protection sleeve carefully inserted to the pin entry point. The orientation is from anterior to posterior in a slight caudal to cranial direction and from lateral to medial following the direction identified by palpation of the iliac crest with the surgeon's free hand, usually at $\sim 20^{\circ}$. An artery clip or the blunt aspect of a K-wire can also be inserted on the lateral inferior aspect of the iliac wing to guide the alignment of the pin. The pin is advanced at low-speed to remain between the medial and lateral cortices as long as possible and can be either uni-cortical or bi-cortical depending on the working length achieved (Figures 2-4). Of note, we have found it easier to put the pins in while standing on the contralateral side of the patient. Image intensifier can be used to guide the direction of the pin; however, we have been very successful in confirming good positioning after both pins have been inserted. A standard AP view of the pelvis is obtained, as well as a modified obturator oblique-outlet view performed in a way to center the pin in a "bull's eye" fashion, often rotating $\sim 20^{\circ}$ from the AP view toward the obturator oblique view, and adding $\sim 10^{\circ} \mathrm{s}$ of outlet view until the pin is centered on the imaging (Figure 5), which confirms that the pin is central in the tuberous region of the iliac bone. The external fixator construct is completed with 2 or 3 carbon-fiber rods connected with pin-to-rod clamps and rod-to-rod clamps, and the pelvis is reduced with a combination of external manipulation or traction, often using the pins as "joysticks." The construct is then tightened. The image intensifier is used with standard AP, inlet and outlet views to assess the quality of the reduction of the anterior and posterior pelvic ring (Figure 6). Procedural time is in the order of $10 \mathrm{~min}$, similar to the subcristal technique (8).

The postoperative care includes regular dry-dressings of the pin sites and observation for any signs of pin site infection. Weight-bearing status is dictated by fracture configuration (Figure 7). Follow-up in the clinic with radiographs occurred at 2, 6, and 12 weeks and 6 months at least until clinical and radiological union with healed surgical wounds or death.

\section{RESULTS}

The cohort of 27 patients included 17 males and 10 females with a mean age of 44.6 years (range 18-80 years) and distributed among all age categories (Figure 8). Demographic data and results are summarized in Table 1. The external fixators have been used as an acute stabilization procedure in 16 patients and as a short-term "definitive" fixation in 10 patients. One patient died on day 14 from the sequelae of his severe traumatic brain injury. In the temporary external fixator group, the external fixator was a temporary stabilization method in the hospital's resuscitation algorithm in patients with unstable pelvic fractures and ongoing hemodynamic instability. These external fixators were then removed during definitive pelvic fixation, at a median 


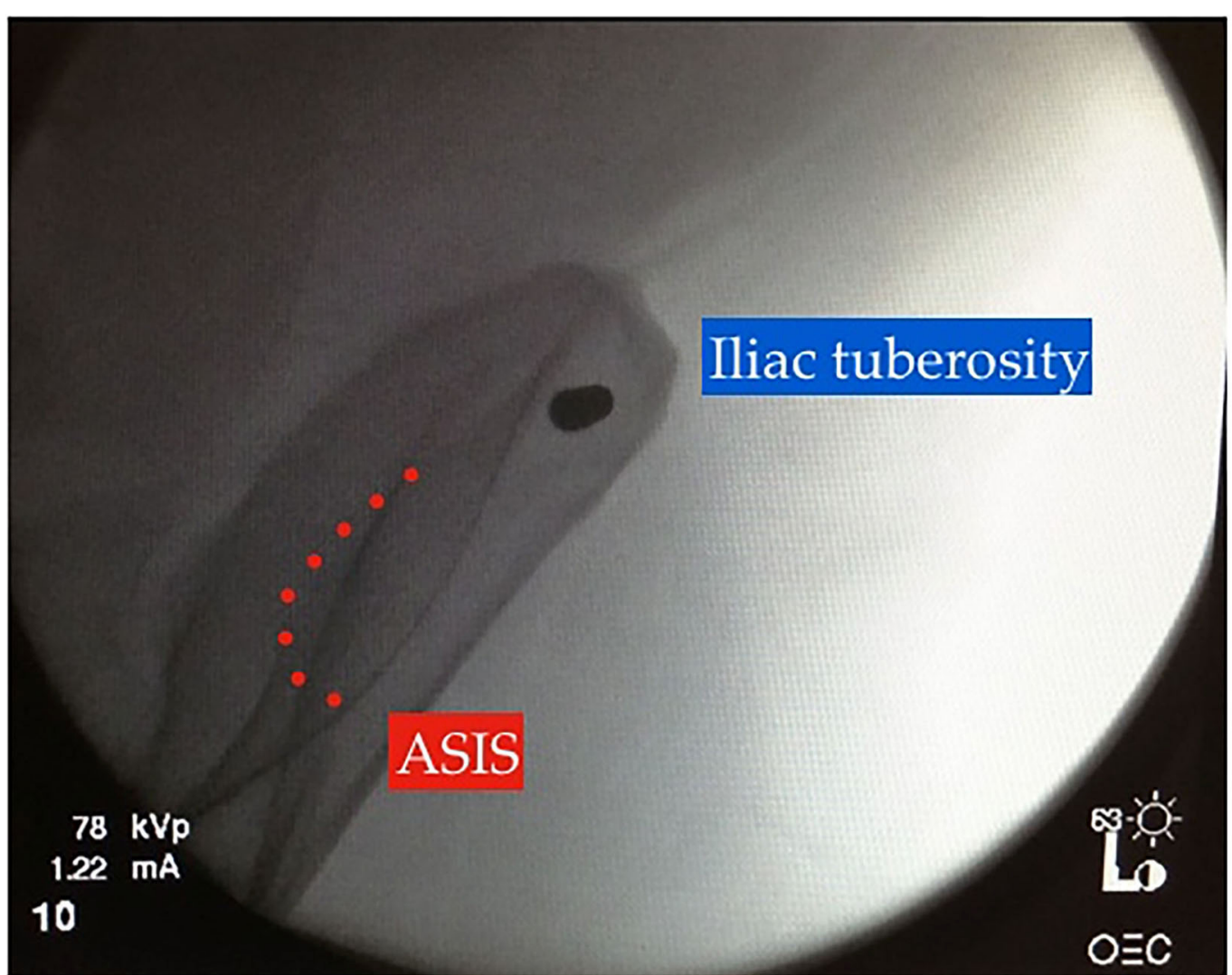

FIGURE 5 | "Bull's eye" view on intraoperative image intensification, modified obturator-oblique view confirming adequate pin position within the iliac tuberosity.

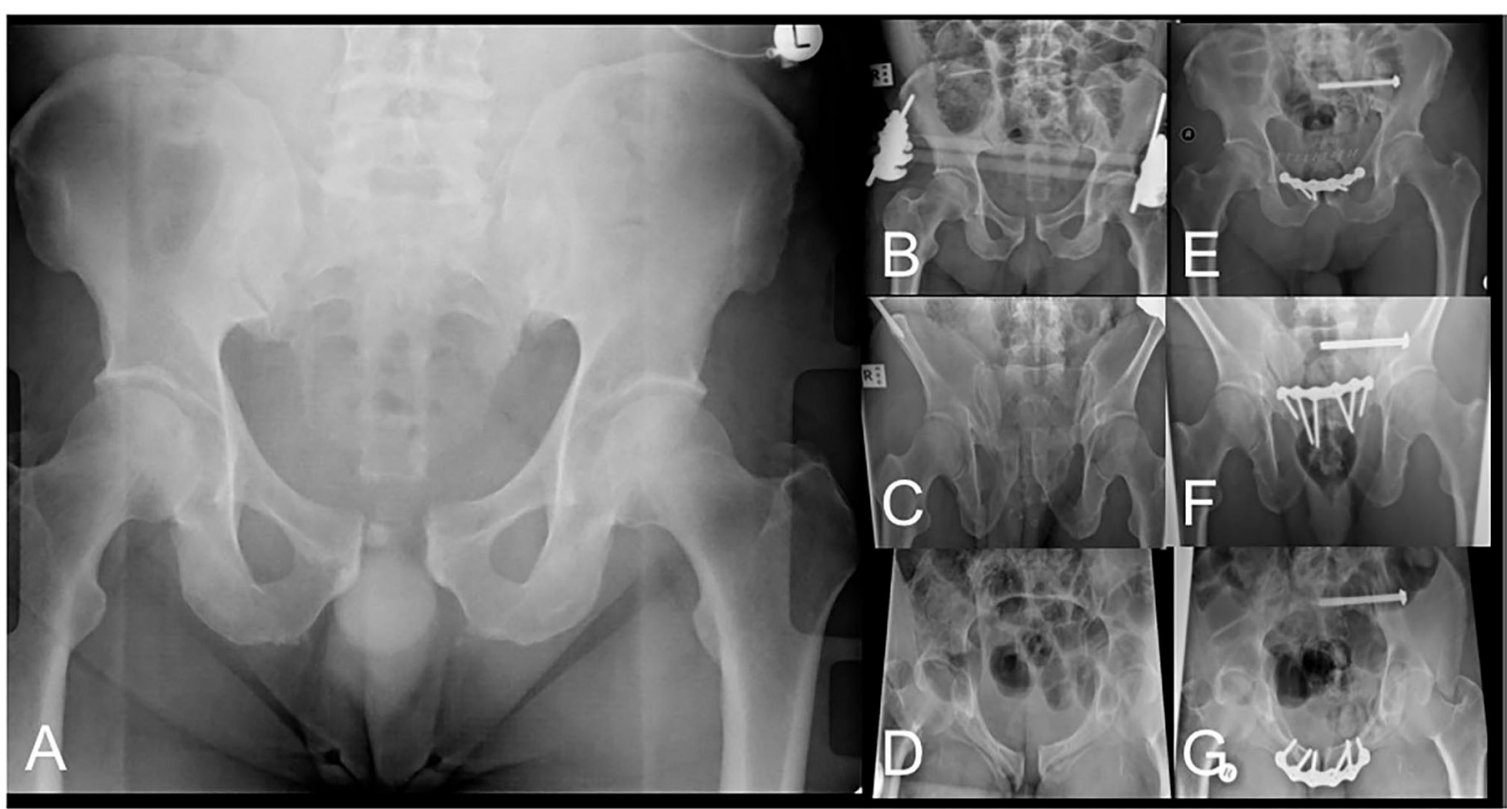

FIGURE 6 | AP pelvis radiograph (A) of pelvic injury classified as left-sided APC2. AP (B), outlet (C) and inlet (D) radiographs after LPEF application demonstrating improved pelvic alignment awaiting definitive surgical management. AP (E), outlet (F) and inlet (G) radiographs after definitive fixation after removal of LPEF. 
time of 2 days postoperatively (IQR $1-3.5$ ). In the latter group, they were removed at a median time of 48 days postoperatively (IQR 37-64), when the fractures were considered healed enough by radiological and clinical criteria for external fixation removal.

The pelvic injuries in these patients were classified, according to the Young and Burgess $(11,12)$ classification based on mechanism of injury, as: 1 LC1, 5 LC2, 13 LC3, 4 APC2, 1 APC3, and 3 VS. Three patients had combined pelvic and acetabular fractures. Two patients had open fractures: one patient sustained an open LC2 fracture, and the patient who died on the 14th day post-injury had an open LC3 pelvic fracture.

The most common cause of injury involved traffic accidents (18/27 patients): motorcycle accidents (seven patients), motor vehicle accidents (five patients), and pedestrian struck by motor vehicles (six patients). The other causes of injury included crush injuries (five patients), fall from a horse (two patients), and fall from own height (two patients). Six patients were transferred from peripheral hospitals. All except for one patient received the external fixator within $24 \mathrm{~h}$ of the injury. The median duration of stay in the hospital was 16 days (IQR 10-31). Nine patients underwent a laparotomy and one patient a thoracotomy. One patient also required a C-clamp (patient T3 with VS pattern combined with right acetabular fracture pattern). One patient received a combination of an LPEF pin and a supra-acetabular pin on the contralateral side because of the particular open fracture configuration (patient F1 with open pelvic fracture LC3).

In the patients for whom the external fixators were temporary, this was converted using various combinations of different surgical fixation methods, including anterior pelvic plating, sacroiliac screws, and posterior pelvic fixation (posterior superior iliac spine entry-point of pedicle-screw fixation system). In the short-term "definitive" treatment group, eight patients were treated with an external fixator in isolation, whereas one patient also received bilateral sacroiliac screw fixation, and one patient received posterior pelvic fixation. The patient who died at 14 days post-injury also required bilateral sacroiliac screw fixation.

In the temporary treatment group, no patients had any complications from this technique. In the short-term "definitive" treatment group, two patients sustained superficial pin site infection (cellulitis and purulent discharge), which resulted in the removal of the external fixator and washout of the wounds at 5 weeks (36 days) and 7 weeks (50 days) after initial application. One other patient had hypergranulating pin sites and a loose external fixator; however, it had functioned well and was removed at 10 weeks (71 days) after application. All the fractures obtained clinical and radiological union in good alignment.

\section{DISCUSSION}

We have described the use of the LPEF acutely in 27 patients with pelvic ring injuries. Numerous studies have documented beneficial results using emergent pelvic external fixation in the resuscitation phase of hemodynamically unstable polytrauma patients with unstable pelvic ring injuries (1-5). However, many different trauma resuscitation protocols exist (13). As $\sim 80 \%$ of cases of hemodynamic instability are related to venous bleeding

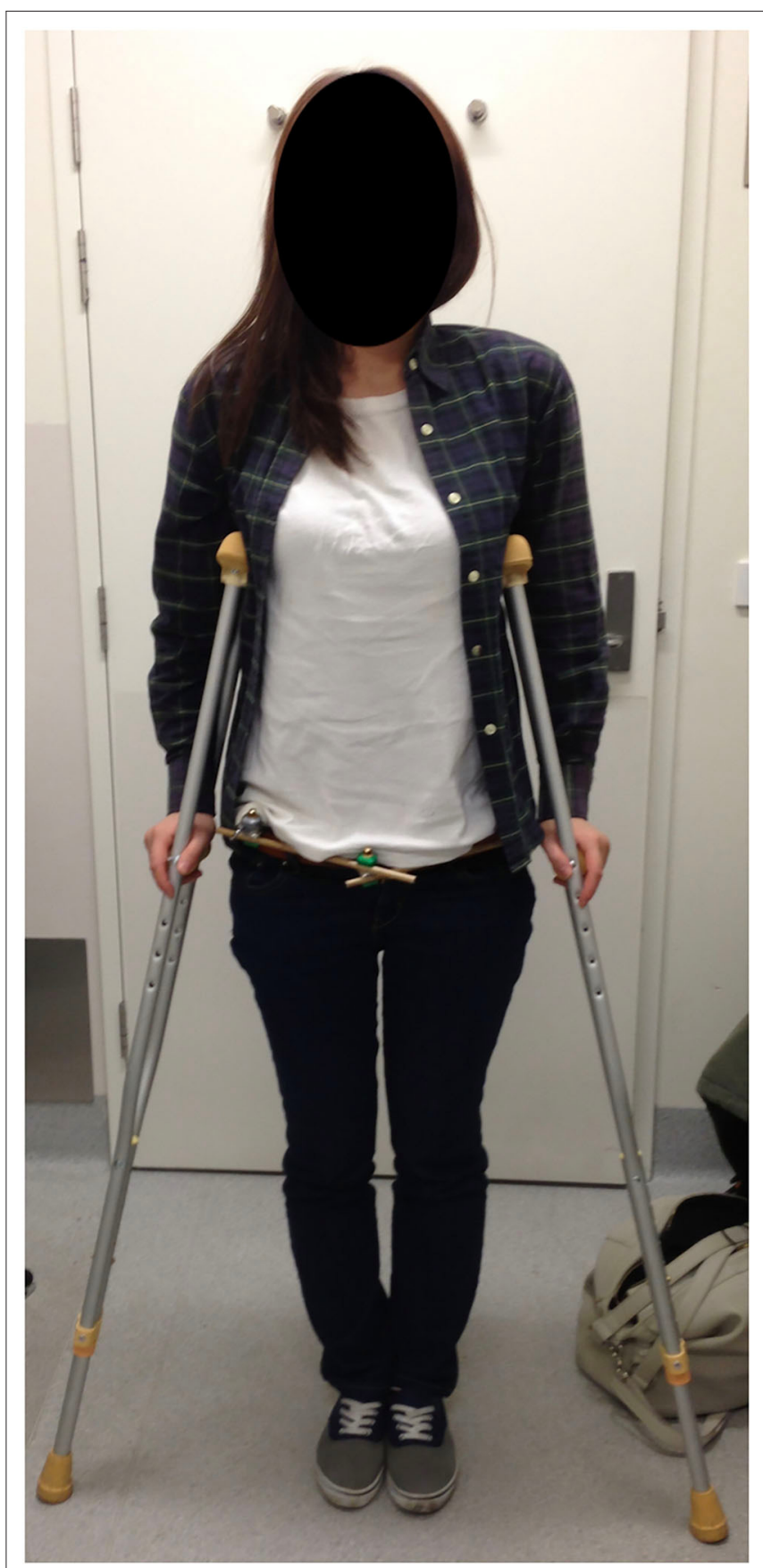

FIGURE 7 | Patient at 2 weeks after LPEF demonstrating ease of clothing and mobility. Weight-bearing dictated by fracture configuration, this specific patient had a left-sided LC1 injury and was allowed full weight-bearing on the right and protected on the left with crutches.

or fracture site bleeding (14), it is crucial to reduce the volume of the pelvis emergently to facilitate clot formation and stop ongoing venous bleeding. Indeed, it has been demonstrated in experimental studies that reduction of open-book pelvic disruptions leads to increased retroperitoneal pressures, and this is believed to contribute to a tamponade effect of venous bleeding (15). This may be achieved by using, for example, a pelvic binder, 


\section{Mean age 44,6 years (range 18-80)}

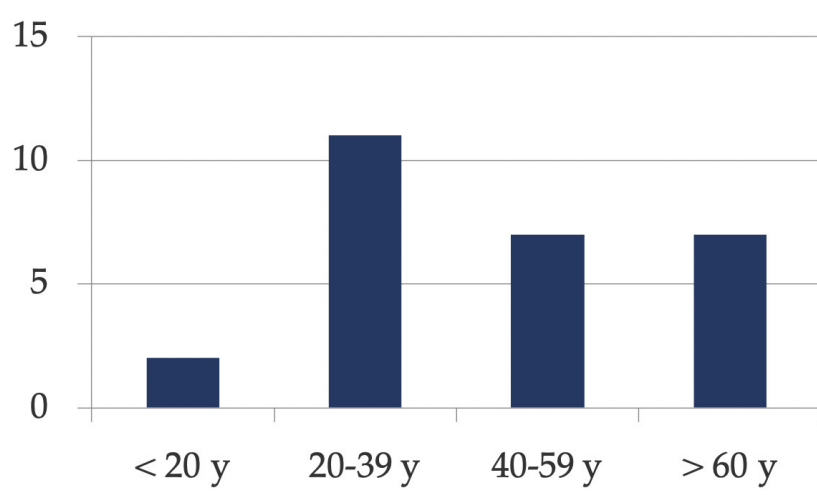

FIGURE 8 | Histogram demonstrating that the LPEF was used in patients of all age categories.

sheet or an external fixation device (including C-clamp). Pelvic packing is another method of creating a tamponade effect to control bleeding, and this may be done in the retroperitoneal space (16). Angioembolization (17) is useful to control arterial bleeding if a blush is visible on a contrast CT scan or in the case of persistent instability after pelvic binder or external fixation application. However, several trauma resuscitation protocols exist, many of which involve pelvic external fixation at some stage. Two different protocols have recently been compared in one study including 348 cases, and there was no difference in mortality, revealing that individualized trauma resuscitation protocols optimizing hospital-available resources are key in treating severely polytraumatized patients (18).

There are currently three recognized sites for pin placement in anterior pelvic external fixation: (1) anterosuperior with pins inserted perpendicular to the iliac crest, (2) supra-acetabular (or anteroinferior) with pins placed from the anterior inferior iliac spine in an anteroposterior direction, and (3) subcristal with pins placed in the anterior superior iliac spine in a direction parallel with the crest (19) (Figure 1). Solomon et al. (8) developed the subcristal approach in response to complications of pin placement in patients referred by peripheral hospitals to their trauma center. Most complications involved failure to correctly place the pin between the inner and outer tables of the ilium, injury to anatomic structures located between the skin and bony entry point, such as the lateral femoral cutaneous nerve, penetration in the hip joint, loss of fixation, and infection. Pin site infection with anterosuperior and supra-acetabular techniques have been reported as high as 20-40 and 25\%, respectively, in definitive treatment cases $(9,10,20)$, and Solomon et al. (8) reported a $20 \%$ incidence of superficial infection in a cohort of 20 patients with subcristal external fixators. Although the entry point of the subcristal technique, the anterior superior iliac spine, is near the lateral femoral cutaneous nerve (21), there were no cases of lateral femoral cutaneous nerve injury in the Solomon et al. (8) series.

The results from the retrospective review of our use of this technique suggest that it may be an alternative to other techniques described; further comparative research of the different techniques would be useful in the future. This technique is quick, and the pins and frame are positioned far from the abdomen, and laparotomy is easily performed in the trauma setting. Our patient cohort is quite heterogeneous, with patients of a wide age range, various pelvic disruption configurations, and various energy levels of the mechanism of injury, and our results suggest that this technique can be used in a very large range of clinical situations. One of the benefits we have found with this technique is the increased distance from the groin, and minimal movement of the skin folds around the pin sites during sitting and hip flexion as demonstrated in Figure 3, in contrast to other techniques. In fact, the two cases of pin site infections in this cohort were at 5 and 7 weeks after LPEF application, at which point the fractures had consolidated enough that the external fixators could be removed safely without any further impact on treatment.

A benefit of the LPEF technique, compared with the subcristal approach, is its ability to remain far away from the surgical field if a Pfennansteil incision were to be required later for anterior pelvic fixation (Figure 2). We believe that we have some added control of the posterior hemipelvis with the LPEF technique compared with the subcristal and iliac wing techniques due to the posterior orientation of the pins. We have also used these lateral posterior pins as temporary indirect reduction aids while undertaking definitive fixation of pelvic fractures. These pins can be used as "joysticks" to control the hemipelvis while reducing anterior pelvic fractures or pubic symphysis disruptions, as well as during percutaneous sacroiliac fixation. It can also be used in an "open" fashion if a lateral window approach is used, for instance, during open sacroiliac reduction.

One patient in the current study received a combination of a lateral posterior pin and a supra-acetabular pin on the contralateral side because of the particular fracture configuration. This is a very good case example demonstrating that several pin entry sites should be mastered by the orthopedic surgeon treating pelvic fractures, as their presentations are quite variable, and one particular technique does not necessarily fit every fracture pattern.

Our study has inherent limitations. As it is a retrospective review, there may be missing data regarding particular complications. However, we have followed up with all the patients in the present study at least until clinical and radiological union or death; therefore, we believe that we have limited the number of omissions in this study. Moreover, the number of patients in the study is limited, particularly in the definitive treatment group, as this study was done to document the initial results and describe the surgical technique of this alternative surgical technique. Furthermore, we have not collected body mass index data for this group of patients, which could influence the risk of infection and ease of surgical technique. Finally, there was no control group. Further studies with a higher number of cases and comparative groups would also be beneficial.

Our results indicate that the lateral posterior pelvic external fixator technique has a low complication rate, at least comparable with previously described techniques, likely explained by the safe anatomical profile of its insertion, inviting further comparative studies. We believe that this technique is very useful in the arsenal 
TABLE 1 | Patients' demographic data and results.

Patient Age at Gender Y-B Mechanism Transfer Days of Days of Days of Converted to Laparotomy Thoracotomy Infection Skin problems Fracture Malposition Loosening Nerve injury hospitalization definitive temporary

\begin{tabular}{llllclll}
\hline D1 & 34 & M & LC3 & MVA & Direct & 23 & \\
D2 & 31 & F & LC2 & MVA & Direct & 31 & 67 \\
D3 & 61 & F & LC3 & $\begin{array}{c}\text { Crush } \\
\text { Transfer }\end{array}$ & 17 & 64 \\
D4 & 31 & F & LC3 & $\begin{array}{c}\text { Pedestrian- } \\
\text { car }\end{array}$ & Transfer & 10 & 52 \\
& & & & Crush & Direct & 7
\end{tabular}

$$
\text { LPEF }
$$

71
67
64
52

\begin{tabular}{|c|c|c|c|c|c|c|c|}
\hline D6 & 44 & $\mathrm{~F}$ & LC1 & MVA & Direct & 10 & 46 \\
\hline D7 & 20 & M & LC3 & $\begin{array}{l}\text { Pedestrian- } \\
\text { truck }\end{array}$ & Direct & 58 & 45 \\
\hline D8 & 72 & $\mathrm{~F}$ & LC3 & $\begin{array}{l}\text { Pedestrian- } \\
\text { car }\end{array}$ & Direct & 39 & 37 \\
\hline D9 & 18 & $\mathrm{~F}$ & LC3 & Fall horse & Direct & 6 & 36 \\
\hline
\end{tabular}

D5 $70 \quad$ M LC3 Crush Direct

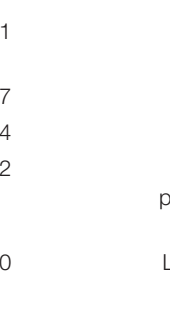

LPEF +
posterior pelvis
fixation
$\mathrm{LPEF}+\mathrm{R}+\mathrm{L}$
$\mathrm{SI}$ screws

Yes
No
No
Yes
No

No No

No Hypergranulation

No No no

No No

No

No No

\begin{tabular}{|c|c|c|c|c|c|c|c|c|c|}
\hline & & No & No & No & No & No & No & No & No \\
\hline 11 & $\begin{array}{l}\text { Posterior pelvis } \\
\text { fixation }\end{array}$ & No & No & No & No & No & No & No & No \\
\hline 5 & $\begin{array}{l}\text { Anterior ORIF } \\
\text { Pfennesteil + L } \\
\text { SI screw }\end{array}$ & No & No & No & No & No & No & No & No \\
\hline 4 & $\begin{array}{c}\text { Anterior ORIF } \\
\text { (bilat Stoppa) + } \\
2 \text { R SI screws }\end{array}$ & Yes & No & No & No & No & No & No & No \\
\hline 4 & $\begin{array}{l}\text { Anterior ORIF } \\
\text { Pfennesteil + } \\
\text { posterior pelvis } \\
\text { fixation }\end{array}$ & No & No & No & No & No & No & No & No \\
\hline 3 & $\begin{array}{l}\text { Anterior ORIF } \\
\text { (bilat Stoppa) }\end{array}$ & No & No & No & No & No & No & No & No \\
\hline
\end{tabular}


TABLE 1 | Continued

Patient Age at Gender Y-B Mechanism Transfer Days of Days of Days of Converted to Laparotomy Thoracotomy Infection Skin problems Fracture Malposition Loosening Nerve hospitalization definitive temporary

injury LPEF LPEF

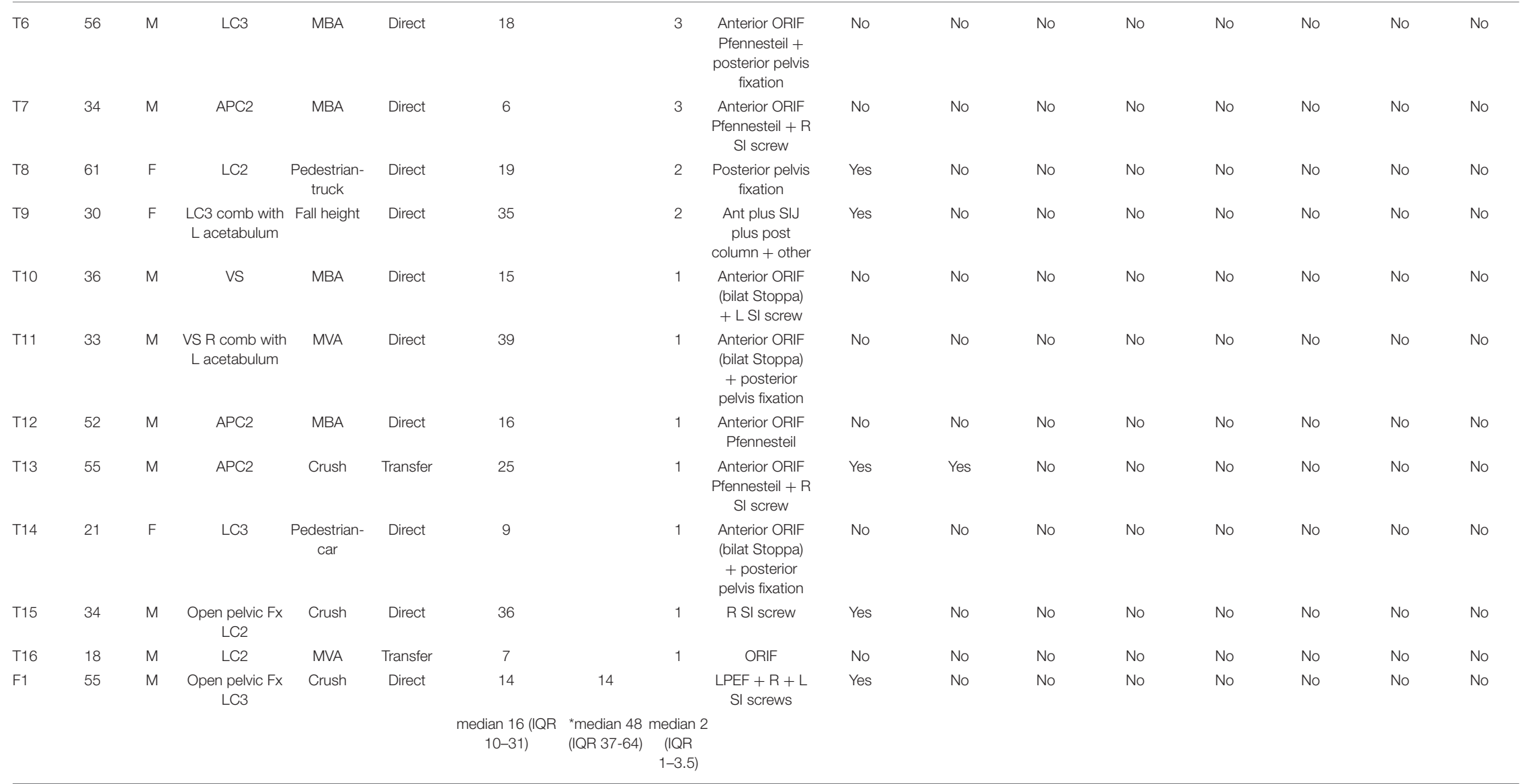

${ }^{*}$ Descriptive statistics for group of definitive LPEF excludes the patient who died on day 14.

$Y-B$, Young and Burgess; $R$, right; L, left; $F x$, fracture; MVA, motor-vehicle accident; MBA, motorbike accident; SI, sacro-liliac; bilat, bilateral; IQR (interquartile range). 
of techniques available to the orthopedic surgeon in treating pelvic fractures.

\section{DATA AVAILABILITY STATEMENT}

The original contributions presented in the study are included in the article/supplementary materials, further inquiries can be directed to the corresponding author/s.

\section{ETHICS STATEMENT}

The studies involving human participants were reviewed and approved by Alfred Hospital Ethics Committee (466/13). Written informed consent for participation was not required

\section{REFERENCES}

1. Burgess AR, Eastridge BJ, Young JW, Ellison PS Jr, Poka A, Bathon GH, et al. Pelvic ring disruptions: effective classification system and treatment protocols. J Trauma. (1990) 30:848-56. doi: 10.1097/00005373-19900700000015

2. Flint L, Babikian G, Anders M, Rodriguez J, Steinberg S. Definitive control of mortality from severe pelvic fracture. Ann Surg. (1990) 211:7067. doi: 10.1097/00000658-199006000-00008

3. Gylling SF, Ward RE, Holcroft JW, Bray TJ, Chapman MW. Immediate external fixation of unstable pelvic fractures. Am J Surg. (1985) 150:7214. doi: 10.1016/0002-9610(85)90416-7

4. Mucha P Jr, Farnell MB. Analysis of pelvic fracture management. J Trauma. (1984) 24:379-86. doi: 10.1097/00005373-198405000-00002

5. Riemer BL, Butterfield SL, Diamond DL, Young JC, Raves JJ, Cottington E, et al. Acute mortality associated with injuries to the pelvic ring: the role of early patient mobilization and external fixation. J Trauma. (1993) 35:671-5; discussion 676-7. doi: 10.1097/00005373-199311000-00003

6. Palmer S, Fairbank AC, Bircher M. Surgical complications and implications of external fixation of pelvic fractures. Injury. (1997) 28:649-53. doi: 10.1016/S0020-1383(97)00141-1

7. Mason WTM, Kahn SN, James CL, Chesser TJS, Ward AJ. Complications of temporary and definitive external fixation of pelvic ring injuries. Injury. (2005) 36:559-604. doi: 10.1016/j.injury.2004.11.016

8. Solomon LB, Pohl AP, Sukthankar A, Chehade MJ. The subcristal pelvic external fixator: technique, results, and rationale. J Orthop Trauma. (2009) 23:365-9. doi: 10.1097/BOT.0b013e3181a2aec3

9. Lindahl J, Hirvensalo E, Böstman O, Santavirta S. Failure of reduction with an external fixator in the management of pelvic ring injuries. J Bone Joint Surg Br. (1999) 81:955-62. doi: 10.1302/0301-620X.81B6.0810955

10. Majeed SA. External fixation of the injured pelvis. The functional outcome. J Bone Joint Surg Br. (1990) 72:612-4. doi: 10.1302/0301-620X.72B4.23 80212

11. Young JW, Burgess AR, Brumback RJ, Poka A. Pelvic fractures: value of plain radiography in early assessment and management. Radiology. (1986) 160:445-51. doi: 10.1148/radiology.160.2.3726125

12. Young JWR, Burgess AR. Radiologic Management of Pelvic Ring Fractures: Systematic Radiographic Diagnosis. Baltimore, MD: Urban \& Schwarzenberg (1987).

13. Hak DJ, Smith WR, Suzuki T. Management of hemorrhage in life-threatening pelvic fracture. JAAOS. (2009) 17:44757. doi: 10.5435/00124635-200907000-00005 for this study in accordance with the national legislation and the institutional requirements. Written informed consent was obtained from the individual(s) for the publication of any potentially identifiable images or data included in this article.

\section{AUTHOR CONTRIBUTIONS}

MR contributed to study design, performed surgeries, and reviewed the manuscript. PN and JS contributed to study design, ethics application, data analysis, and writing of the manuscript. All authors contributed to the article and approved the submitted version.

14. Smit WR, Ziran BH, Morgan SJ. Fractures of the Pelvis and Acetabulum. New York, NY. (2007). p. 317. doi: 10.3109/9781420016383

15. Grimm MR, Vrahas MS, Thomas KA. Pressure-volume characteristics of the intact and disrupted pelvic retroperitoneum. J Trauma. (1998) 44:4549. doi: 10.1097/00005373-199803000-00006

16. Cothren CC, Osborn PM, Moore EE, Morgan SJ, Johnson JL, Smith WR. Preperitoneal pelvic packing for hemodynamically unstable pelvic fractures: a paradigm shift. J Trauma. (2007) 62:834-9; discussion 83942. doi: 10.1097/TA.0b013e31803c7632

17. Fangio P, Asehnoune K, Edouard A, Smail Nadia, Benhamou D. Early embolization and vasopressor administration for management of lifethreatening hemorrhage from pelvic fracture. J Trauma. (2005) 58:97884. doi: 10.1097/01.TA.0000163435.39881.26

18. Gabbe BJ, de Steiger R, Esser M, Bucknill A, Russ MK, Cameron PA. Predictors of mortality following severe pelvic ring fracture: results of a population-based study. Injury. (2011) 42:985-91. doi: 10.1016/j.injury.2011.06.003

19. Solomon LB, Pohl AP, Chehade MJ, Malcolm AM, Howie DW, Henneberg M. Surgical Anatomy for pelvic fixation. Clin Anatomy. (2008) 21:67482. doi: $10.1002 / \mathrm{ca} .20697$

20. Cole PA, Gauger EM, Anavian J, Ly TV, Morgan RA, Heddings AA. Anterior pelvic external fixator versus subcutaneous internal fixator in the treatment of anterior ring pelvic fractures. J Orthop Trauma. (2012) 26:26977. doi: 10.1097/BOT.0b013e3182410577

21. Grothaus MC, Holt M, Mekhail AO, Ebraheim NA, Yeasting RA. Lateral femoral cutaneous nerve: an anatomic study. Clin Orthop Relat Res. (2005) 437:164-8. doi: 10.1097/01.blo.0000164526.08610.97

Conflict of Interest: PN reports payment for development of educational presentations from DePuy Synthes and travel/accommodations/meeting expenses from Stryker, both unrelated to this article.

The remaining authors declare that the research was conducted in the absence of any commercial or financial relationships that could be construed as a potential conflict of interest.

Copyright (c) 2021 Russ, Navarre and Stevens. This is an open-access article distributed under the terms of the Creative Commons Attribution License (CC BY). The use, distribution or reproduction in other forums is permitted, provided the original author(s) and the copyright owner(s) are credited and that the original publication in this journal is cited, in accordance with accepted academic practice. No use, distribution or reproduction is permitted which does not comply with these terms. 